Open Access

\title{
Molecular cloning and expression analysis of adiponectin and its receptors (AdipoR1 and AdipoR2) in the hypothalamus of the Huoyan goose during different stages of the egg-laying cycle
}

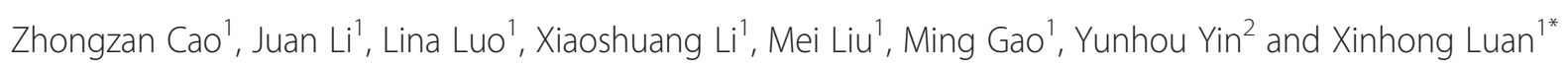

\begin{abstract}
Background: Adiponectin and its receptors (AdipoR1 and AdipoR2) are novel endocrine systems that act at various levels to regulate metabolic homeostasis and reproductive processes. We cloned and characterized the cDNA of adiponectin and its receptors from the hypothalamus of the Huoyan goose to reveal the influence of these factors on the process of goose egg-laying. We also determined the mRNA and protein expression profiles during different stages of the egg-laying cycle.

Methods: Hypothalamus tissues were obtained from 36 Huoyan geese in the pre-laying, early-laying, peak-laying, and ceased periods. The cDNA sequences of goose adiponectin and its receptors (AdipoR1 and AdipoR2) were cloned and characterized using the 5'-RACE and 3'-RACE methods. Multiple alignments and phylogenetic analyses of the deduced amino acid sequence were conducted using bioinformatics tools. The expression profiles of mRNA and protein in the hypothalamus during the pre-laying, early-laying, peak-laying and ceased periods were examined using real-time PCR (qRT-PCR) and Western blotting techniques.

Results: The CDNA of adiponectin, AdipoR1 and AdipoR2 consisted of 738, 1131 and 1161 bp open reading frame encoding 245, 376 and 386 amino acids, respectively. The deduced amino acid sequence of goose adiponectin, as well as AdipoR1 and AdipoR2 showed a closer genetic relationship to the avian species than to other mammal species. The expression level of adiponectin mRNA and protein increased from the pre-laying period to the peak-laying period, reached its peak in the peak-laying period, and then decreased during the ceased period. Conversely, the expression levels of AdipoR1 and AdipoR2 mRNA and protein decreased in the early-laying period, peak-laying period, and ceased period compared with the pre-laying period.
\end{abstract}

Conclusions: This study is the first to obtain full-length cDNA sequences of goose adiponectin and the genes of its receptors from the hypothalamus, and demonstrate that the egg-laying cycle affects the expression of the goose adiponectin system. Our results suggest the potential role of adiponectin as a key neuromodulator of reproductive functions.

Keywords: Huoyan goose, Adiponectin, Adiponectin receptor, Hypothalamus, Egg-laying cycle

\footnotetext{
*Correspondence: xhluan@163.com

${ }^{1}$ College of Animal Science and Veterinary Medicine, Shenyang Agricultural

University, Shenyang 110866, China

Full list of author information is available at the end of the article
} 


\section{Background}

Adiponectin is one of adipocytokine hormones secreted primarily by adipocytes. It belongs to a family of proteins that contain sequences homologous to the C1q globular domain. Adiponectin consists of an N-terminal collagenous domain and C-terminal globular domain $[1,2]$. The biological activity of this hormone is mediated via two distinct receptors, termed adiponectin receptor 1 (AdipoR1) and adiponectin receptor 2 (AdipoR2). Both receptors contain seven transmembrane domains with an extracellular carboxyl terminus and an intracellular amino terminus that are structurally and functionally distinct from G protein-coupled receptors. AdipoR1 shows high affinity for the globular form of adiponectin, and AdipoR2 has an intermediate binding affinity for both full-length and globular species [3, 4]. Activation of these cell membrane bound receptors can then activate the AMP activated protein kinase (AMPK), peroxisome proliferator activated receptor alpha (PPAR $\alpha$ ) and mitogen activated protein kinase (MAPK) [5]. Studies have suggested that the adiponectin, AdipoR1 and AdipoR2 genes are expressed in multiple tissues including the anterior pituitary gland, hypothalamus, testis and ovary in mammals and Avian [6, 7].

Adiponectin plays a dominant role in lipid and carbohydrate metabolism, stimulates fatty acid oxidation, decreases plasma triglycerides, and improves glucose metabolism by increasing insulin sensitivity [3]. Adiponectin also seems to be an important factor linking the regulation of reproductive processes [8]. Evidence has indicated that adiponectin influences the reproductive system by exerting central or peripheral effects on the hypothalamus-pituitary-gonadal (HPG) axis. The adiponectin receptors (AdipR1 and AdipR2) are expressed in mouse GT1-7 cells derived from hypothalamus neurons, and adiponectin inhibits $\mathrm{GnRH}$ secretion through the activation of AMP-activated protein kinase (AMPK) [9]. The expression of adiponectin and its receptors is also responsible for $\mathrm{GnRH}$ secretion in porcine hypothalamic structures including the medio-basal hypothalamus $(\mathrm{MBH})$, preoptic area (POA) and median eminence (SME)[10]. Adiponectin could influence oxytocin-secreting neuron excitability and enhanced oxytocin secretion in the paraventricular nucleus of the hypothalamus (PVN) [11]. In addition, adiponectin seems to be involved in the autocrine/paracrine control of pituitary gonadotrophs and the secretion of LH, GH and FSH [12-14]. It was also described as a factor affecting ovarian steroidogenesis and oocyte maturation [15-17]. Therefore, the relation between expression in the hypothalamus, pituitary and gonadal adiponectin systems and the reproductive cycle has been investigated in porcine, cow, and chicken $[15,18$, 19]. These results demonstrated that the adiponectin system may affect reproductive functions by controlling the hypothalamic-pituitary-gonadal axis and that the expression of adiponectin and the adiponectin receptors is dependent on the endocrine status of animals.

In poultry, the reproductive endocrine system and reproductive activity are strictly controlled by the hypothalamic-pituitary-gonadal axis [20]. The hypothalamus regulate reproduction by releasing neurohormones (gonadotropin-releasing hormones, $\mathrm{GnRH}$ ) to the pituitary gland, and then the pituitary gland synthesizes and releases gonadotropins (luteinizing hormone, $\mathrm{LH}$; folliclestimulating hormone, FSH), which in turn act on the gonads to stimulate gametogenesis (spermatogenesis, oogenesis) and sex steroid hormones secretion (androgens, oestrogens, and progesterone). To our knowledge, there are no other reports regarding the expression changes of adiponectin, AdipoR1 and AdipoR2 in the hypothalamicpituitary-gonadal axis of the goose during different egglaying stages. Therefore, the current study was undertaken to clone the full-length cDNA of Huoyan goose adiponectin and its receptors by RACE (rapid amplification of cDNA ends), and identify its sequence characteristics. Subsequently, we aimed to determine the gene and protein expression profiles of these genes in the hypothalamus of the Huoyan goose during pre-laying, early-laying, peak-laying and ceased periods, with the use of real-time PCR and western blotting. These results provide a more complete understanding of the action of the adiponectin system with respect to the process of geese egg-laying.

\section{Methods}

\section{Animal and tissue collection}

Thirty-six Huoyan geese were selected randomly from two hundred geese on the Liaoning Huoyan goose stock breeding farm and raised according to the farm program. During the experiment, geese were fed ad libitum with rice grain and were supplemented with green grass or water plants whenever possible. Feed was given during the daytime when the geese were released into an open area outside the house. Huoyan geese become sexually mature at approximately 7 months of age and reach the peak egg-laying stage in the following year. In the current study, goslings were purchased in the fall of the year and become sexually mature during the summer of the following year. Nine geese were killed by exsanguination at the age of 6 months (pre-laying period), 9 months (early laying period), 12 months (peak-laying period), and 15 months (ceased period). The hypothalamus were quickly dissected, frozen in liquid nitrogen, and stored at $-80{ }^{\circ} \mathrm{C}$ until total RNA extraction and protein were prepared. All experimental procedures were reviewed and approved by the animal welfare committee of the College of Animal Science and Veterinary Medicine of Shenyang Agricultural University (No. 2011036) and performed in accordance with the Regulations for 
the Administration of Affairs Concerning Experimental Animals (China, 1988) and EU Directive 2010/63/EU for animal experiments.

\section{Cloning and sequencing of goose adiponectin, AdipoR1 and AdipoR2}

Total RNA was extracted using Trizol reagent (Invitrogen Corporation, Carlsbad, CA) following the manufacturer's protocol. The quality of the RNA was determined by agarose gel electrophoresis and NanoDrop 8000 spectrophotometry (NanoDrop, Thermo Scientific). One microgram of RNA was reversely transcribed to cDNA using a PrimeScript ${ }^{\oplus} \mathrm{R}$ reagent Kit (TaKaRa, Dalian, China) in a total volume of $20 \mu \mathrm{l}$ with $4.0 \mu \mathrm{l}$ of $5 \times$ PrimeScript ${ }^{\circledR}$ Buffer, $1.0 \mu \mathrm{l}$ of PrimeScript ${ }^{\circledR}$ RT Enzyme Mix, $1.0 \mu \mathrm{l}$ of Random 6 mers, $1.0 \mu \mathrm{l}$ of oligo $(\mathrm{dT})_{18}$ Primer and $9.0 \mu \mathrm{l}$ of RNase Free $\mathrm{H}_{2} \mathrm{O}$. Thermal cycling was performed for $15 \mathrm{~min}$ at $37^{\circ} \mathrm{C}$, and then $5 \mathrm{~s}$ at $85^{\circ} \mathrm{C}$. RT products were stored at $20{ }^{\circ} \mathrm{C}$ prior to RT-PCR. Specific PCR primer pairs used to amplify regions of the adiponectin, AdipoR1 and AdipoR2 cDNA sequences were designed by using Primer Premier 6.0 (Primer Biosoft International, Palo Alto, California, USA) according to the mRNA sequence of the Gallus gallus adiponectin AdipoR1 and Anas platyrhynchos AdipoR2 genes (NM_206991.1, NM_00103 1027.1 and XM_005010210.1). All of the primer pairs were synthesized commercially by Sangon Biotech Co., LTD (Shanghai, China). The primers are listed in Table 1. The $50 \mu$ reaction consisted of $1 \mu \mathrm{l}$ of cDNA, $8 \mu \mathrm{l}$ of deoxynucleoside triphosphate $\mathrm{Mix}(2.5 \mathrm{mmol} / \mathrm{L}$ each dATP, dGTP, dCTP and dTTP), $2 \mu$ l of each primer $(10 \mu \mathrm{mol} / \mathrm{l}), 5 \mu \mathrm{l}$ of $10 \times \mathrm{LA}$ PCR Buffer, $0.5 \mu \mathrm{l}$ of $5 \mathrm{U} / \mu \mathrm{L}$ LA $\operatorname{Taq}^{\mathrm{Tm}}$ (TaKaRa, Dalian, China), and $31.5 \mu \mathrm{l}$ sterile MilliQ water. PCR conditions for amplification of adiponectin, AdipoR1, and AdipoR2 were 35 cycles consisting of denaturing at $94{ }^{\circ} \mathrm{C}$ for $30 \mathrm{~s}$, specific annealing for $30 \mathrm{~s}$, and extension at $72{ }^{\circ} \mathrm{C}$ for $90 \mathrm{~s}$ with an initial denaturing step at $94{ }^{\circ} \mathrm{C}$ for $5 \mathrm{~min}$ and a final extension step at $72{ }^{\circ} \mathrm{C}$ for $10 \mathrm{~min}$. The annealing temperatures were 55 , 60 , and $50{ }^{\circ} \mathrm{C}$ for adiponectin, AdipoR1, and AdipoR2,

Table 1 Primers used in this study

\begin{tabular}{|c|c|c|}
\hline Primers purpose & Primer name & Primer sequence $\left(5^{\prime}-3^{\prime}\right)$ \\
\hline \multirow[t]{6}{*}{ RT-PCR } & Adiponectin-F & TCCTCCTITGCTCACTGCT \\
\hline & Adiponectin-R & CGGCCTTGTCCTTCTTGTA \\
\hline & AdipoR1-F & AGGAGGAAGTTGTCCGTGTG \\
\hline & AdipoR1-R & CTTCGAGTCCGTAGCGAAAC \\
\hline & AdipoR2-F & TCAAGAGGAGGCAGGATAT \\
\hline & AdipoR2-R & GGAACCAGATGTCACACTT \\
\hline \multirow[t]{6}{*}{$3^{\prime}-$ RACE } & Adiponectin-GSP3 & AGAACCACTACGACGCCAGCACCG \\
\hline & Adiponectin-NGSP3 & ATGTACTACTTCGCCTACCACCTGACG \\
\hline & AdipoR1-GSP3 & CCACCATGCACTTCACCATCGCCGA \\
\hline & AdipoR1-NGSP3 & GCATCAGATCTTCCATGTGCTCGTG \\
\hline & AdipoR2-GSP3 & CCTGGGCATCGCAGCCATAATTGTC \\
\hline & AdipoR2-NGSP3 & AGATAGGCTGGCTGGCACTCATGGC \\
\hline \multirow[t]{4}{*}{$5^{\prime}-\mathrm{RACE}$} & Adiponectin-GSP5 & CATCTITCCCGTCCCTGC \\
\hline & Adiponectin-NGSP5 & CGGGCTGGGGATCTGGAG \\
\hline & AdipoR1-GSP5 & AGCGCCCTTCCCACACCTTA \\
\hline & AdipoR1-NGSP5 & CATCTTCTCCATGGCGTGGT \\
\hline \multirow[t]{2}{*}{ RACE } & UPM-Long & CTAATACGACTCACTATAGGGCAAGCAGTGGTATCAACGCAGAGT \\
\hline & UPM-Short & CTAATACGACTCACTATAGGGC \\
\hline \multirow[t]{6}{*}{ Real-time PCR } & Adiponectin-S & AACGAGCAGAACCACTAC \\
\hline & Adiponectin-A & CGCCTTGTCCTTCTTGTA \\
\hline & AdipoR1-S & AAGTTGGATTATTCAGGAA \\
\hline & AdipoR1-A & AATGGAGAGGTAGATGAG \\
\hline & AdipoR2-S & ATACTGAACAAGGCCACTATTT \\
\hline & AdipoR2-A & CACCTGAATGCCTTACTCTC \\
\hline \multirow[t]{2}{*}{ Internal control } & $18 \mathrm{~S}$ rRNA-S & CGGACAGGATTGACAGATTGAG \\
\hline & $18 \mathrm{~S}$ rRNA-A & GCCAGAGTCTCGTTCGTTAT \\
\hline
\end{tabular}


respectively. The PCR products were gel-purified and ligated into the pMD-18-T vector (TaKaRa, Dalian, China), transformed into the competent E. coli DH5 $\alpha$ competent cell. Positive clones containing the expected-size inserts were screened by colony PCR and then sequenced by Sangon Biotech Co., LTD, and its characteristics were determined using Basic Local Alignment Search Tool (nBLAST) at http://blast.ncbi.nlm.nih.gov/Blast.cgi.

Based on the partial cDNA sequences of adiponectin, AdipoR1 and AdipoR2 obtained from the above RT-PCR reaction, specific primers were designed to amplify the full-length cDNA sequence of goose adiponectin, AdipoR1 and AdipoR2 (primers shown in Table 1) using the SMARTer $^{\mathrm{rm}}$ RACE cDNA Amplification kit (Clontech Laboratories, CA, USA) according to the manufacturer's instructions. The 3'- and 5'-end cDNA templates were synthesized using the 3'-CDS Primer A and 5'-CDS Primer A provided in the kit. Nested PCR was used in the 3'-RACE analysis. The first-round of PCR was performed in a total volume of $50 \mu \mathrm{l}$ containing $2.5 \mu \mathrm{l}$ of the first strand 3'- end cDNA template, $5.0 \mu \mathrm{l}$ of $10 \times$ Advantage 2 PCR buffer, $1.0 \mu \mathrm{l}$ of $10 \mathrm{mM}$ dNTP Mix, $1.0 \mu \mathrm{l}$ of $10 \mu \mathrm{M}$ gene-specific primer GSP3, $5.0 \mu \mathrm{l}$ of $10 \times$ Universal Primer Mix (UPM; Clontech, USA), $34.5 \mu \mathrm{l}$ of sterile deionized water, and $1.0 \mu \mathrm{l}$ of $50 \times$ Advantage 2 Polymerase Mix (Clontech, USA). Then, $1 \mu \mathrm{l}$ of the PCR product was diluted to $1: 50$ and subsequently amplified with the NGSP3 and UPM as described above. For the 5' RACE, a 5'- end cDNA template, SMARTer ${ }^{\text {ru }}$ cDNA kit UPM and the gene-specific primer GSP5 were used for the first-round PCR. These amplified products were then subjected to a second round of nested PCR with the UPM and NGSP5. PCR amplification conditions for 3' and 5 ' RACE were as follows: 5 cycles at $94{ }^{\circ} \mathrm{C}$ for $30 \mathrm{~s}$ and $72{ }^{\circ} \mathrm{C}$ for $3 \mathrm{~min} ; 5$ cycles at $94{ }^{\circ} \mathrm{C}$ for $30 \mathrm{~s}, 70{ }^{\circ} \mathrm{C}$ for $30 \mathrm{~s}$, and $72{ }^{\circ} \mathrm{C}$ for $3 \mathrm{~min} ; 25$ cycles at $94{ }^{\circ} \mathrm{C}$ for $30 \mathrm{~s}$, $68{ }^{\circ} \mathrm{C}$ for $30 \mathrm{~s}$, and $72{ }^{\circ} \mathrm{C}$ for $3 \mathrm{~min}$; a final extension for $10 \mathrm{~min}$ at $72{ }^{\circ} \mathrm{C}$; and then cooled to $4{ }^{\circ} \mathrm{C}$.

The final PCR products were gel-purified and ligated into the pMD-18-T vector (TaKaRa, Dalian, China) and then transformed into the E. coli DH5 $\alpha$ competent cell. Positive clones containing the expected-size inserts were screened using colony PCR and then sequenced by Sangon Biotech Co., LTD.

\section{Bioinformatic analysis}

The data of DNA sequences were edited and analysed using Lasergene 7.0 software (DNA Star Inc., Madison, USA), and similarity analyses of nucleotide and protein sequences were carried out using the BLAST program from the NCBI (http://blast.ncbi.nlm.nih.gov/Blast.cgi). The open reading frame (ORF) was obtained using the ORF finder (http://www.ncbi.nlm.nih.gov/gorf/gorf.html), and the coding region sequences were translated into amino acid sequences using the sequence manipulation suite (SMS) tool (http://www.bio-soft.net/sms/index.html). The homologous conserved domains were identified using SMART (Simple Modular Architecture Research Tool, http://smart.embl-heidelberg.de). The molecular weight and isoelectric point of this predicted protein were analysed using the ExPASy ProtParam tool (http://web.expasy.org/protparam/). The PSORT II web-based program (http://psort.hgc.jp/form2.html) was used to predict the subcellular distribution of these proteins. The presence of transmembrane regions, phosphorylation sites, Nglycosylation sites and the secondary structure of these proteins were predicted using the TMHMM, version 2.0; NetPhos, version 2.0; NetNGlyc, version 1.0; and SOPMA web-based programs, respectively. Multiple alignments of the adiponectin, AdipoR1, and AdipoR2 sequences were performed with the ClustalX2 program [21] and the phylogenetic tree was constructed using the neighbour-joining (NJ) methods (bootstrap phylogeny test, 1000 replicates) with the MEGA 4.0 program [22].

\section{Quantitative Real-time RT-PCR}

To evaluate changes in the gene expression of adiponectin, AdipoR1, and AdipoR2 in hypothalamus of Huoyan goose during different stages of the egg-laying cycle, quantitative real-time RT-PCR (qRT-PCR) was performed. The information for the primers used for qRTPCR is listed in Table 1. Total RNA was extracted using TRIzol Reagent (Invitrogen Corporation, Carlsbad, CA) according to the manufacturer's instructions. The concentration and purity of the RNA were measured as described above. Two micrograms of total RNA was reverse transcribed using PrimerScript ${ }^{\circ}$ RT reagent Kit (TaKaRa, Dalian, China). Real-time PCR was carried out on the Bio-Rad iQ5 Real-time PCR Detection System and software (BIO-RAD, California, USA). Each $25 \mu \mathrm{l}$ reaction volume contained $1 \mu \mathrm{l} 10 \mu \mathrm{M}$ (each) forward and reverse primers, $12.5 \mu \mathrm{l} 2 \times \mathrm{SYBR}^{\circ}$ Premix Ex Taq ${ }^{\mathrm{Tm}}$ II (Takara, Dalian, China), and $2 \mu \mathrm{l}$ cDNA products, and the final volume was adjusted using PCR-water. The following PCR program was used for amplification: $15 \mathrm{~min}$ at $95{ }^{\circ} \mathrm{C}, 40$ cycles of denaturation at $95^{\circ} \mathrm{C}$ for $10 \mathrm{~s}$ and annealing and extension at $60{ }^{\circ} \mathrm{C}$ for $30 \mathrm{~s}$. The $18 \mathrm{~S}$ rRNA was selected as an internal reference gene and the expression level of $18 \mathrm{~S}$ rRNA was used to normalize the qRT-PCR results for each gene. Negative controls without a cDNA template were included in this experiment. Standard curve testing was performed using a series of 10-fold diluted samples, respectively, for each gene. The slopes of standard curves and PCR efficiency for these genes were calculated to confirm the preciseness and validity of the RT-qPCR data. Melting curves were analysed to ensure that a single PCR product was amplified 
for each pair of primers. Product purity was confirmed by electrophoresis. All samples were amplified in triplicate. Threshold and Ct (threshold cycle) values were determined automatically by the Bio-Rad iQ5 Real-time PCR Detection software using default parameters. The relative levels of expression for adiponectin, AdipoR1, and AdipoR2 were calculated relative to $18 \mathrm{~S}$ rRNA using the $2^{-\Delta \Delta \mathrm{Ct}}$ method [23].

\section{Western blotting analysis}

Protein samples of hypothalamus tissues from the prelaying, early-laying, peak-laying, and ceased groups were extracted and determined using kits according to the manufacturer's instructions (Applygen Co., LTD. Beijing, China). Equivalent amounts of total protein were subjected to $12 \%$ SDS-PAGE and then transferred to a nitrocellulose membrane. After blocking for $1 \mathrm{~h}$ at $37^{\circ} \mathrm{C}$, the membranes were incubated separately with rabbit Anti-Adiponectin antibody (bs-0471R, Beijing Biosynthesis Biotechnology Co., LTD), rabbit Anti-Adiponectin Receptor 1 antibody (bs-0610R, Beijing Biosynthesis Biotechnology Co., LTD), and rabbit anti-Adiponectin receptor 2 antibody (bs-0611R, Beijing Biosynthesis Biotechnology Co., LTD) overnight at $4{ }^{\circ} \mathrm{C}$. The membranes were subsequently incubated with HRP-conjugated goat anti-rabbit antibody (bs-0295G-HRP, Beijing Biosynthesis Biotechnology Co., LTD) for $1 \mathrm{~h}$ at $37{ }^{\circ} \mathrm{C}$. Finally, the bands were captured using a MicroChemi4.2 imaging system (DNR Bio-imaging Systems, Jerusalem, Israel), and densitometry analysis of protein bands was performed using GelQuant software (DNR Bio-imaging Systems, Jerusalem, Israel). $\beta$-actin (sc-47778, Santa Cruz Biotechnology, USA) was used as a reference protein to ensure equal loading. Triplicate experiments were performed for each sample.

\section{Statistical analysis}

The mRNA level or protein abundance of adiponectin, AdipoR1, and AdipoR2 in the pre-laying period was assigned a value of 1 . All data were analysed using SPSS 16.0 for Windows (SPSS Inc. Chicago, Illinois, USA). The data were analysed by one-way ANOVA, followed by a Tamhane's T2 post hoc test. The results are expressed as the mean \pm SEM. $\mathrm{P}<0.05$ was considered to be statistically significant.

\section{Results}

\section{Cloning and characteristics of the adiponectin, AdipoR1 and AdipoR2 CDNA}

The full-length cDNA sequences of the goose adiponectin, AdipoR1 and AdipoR2 genes were obtained as described above and deposited in GenBank (GenBank Accession No. KP993199, KP993200, and KP993201), respectively. Adiponectin is $2221 \mathrm{bp}$ in length and consists of a 55 bp 5' UTR, a 738 bp ORF encoding 245 amino acids (Additional file 1: Figure S1), and a 1428 bp 3' UTR. According to the prediction from ProtParam, the molecular mass of the goose adiponectin protein is $26.5416 \mathrm{kDa}$, and the theoretical isoelectric point is 5.19 . Under the analysis of the deduced amino acid sequence by the SMART program, adiponectin contained one C1Q domain (Complement component C1q domain) from amino acid residues 105 to 241. The subcellular distribution of the adiponectin protein was predicted to be $66.7 \%$ extracellular, $22.2 \%$ vacuolar, and $11.1 \%$ endoplasmic reticulum. Eleven putative phosphorylation sites were identified in the adiponectin protein, which included five serine residues (Ser75, Ser115, Ser173, Ser205, and Ser231), three threonine residue (Thr33, Thr84, and Thr119), and three tyrosine residues (Tyr108, Tyr142, and Tyr224). The secondary structure of the adiponectin protein was predicted to consist of $2.45 \% \alpha$ helix, $27.76 \%$ extended strand, $9.39 \% \beta$-turn, and $60.41 \%$ random coil. No N-glycosylation sites were found in the transmembrane domain.

AdipoR1 contains 1465 bp with a 206 bp 5' UTR, an 1131 bp ORF encoding 376 amino acids (Additional file 2: Figure S2), and a 128 bp 3' UTR. The predicted molecular mass of the goose AdipoR1 protein is $42.2623 \mathrm{kDa}$, and the theoretical isoelectric point is 6.92. The subcellular distribution of the AdipoR1 protein was predicted to be $55.6 \%$ in endoplasmic reticulum, $33.3 \%$ in plasma membrane, and $11.1 \%$ in mitochondria. As in mammals, seven transmembrane domains were found from the amino acids residues 136 to 158,173 to 195,208 to 227,237 to 256, 269 to 288,298 to 320 , and 333 to 355 . Ten putative phosphorylation sites were identified in the AdipoR1 protein, which included five serine residues (Ser48, Ser198, Ser202, Ser206, and Ser211), two threonine residues (Thr261 and Thr267), and three tyrosine residues (Tyr86, Tyr110, and Tyr210). The secondary structure of the AdipoR1 protein was predicted to consist of $38.3 \% \alpha$-helix, $21.28 \%$ extended strand, $9.04 \% \beta$-turn, and $31.38 \%$ random coil. No N-glycosylation site was found.

AdipoR2 is $3820 \mathrm{bp}$ in length and consists of a $41 \mathrm{bp}$ 5' UTR, a 1161 bp ORF encoding 386 amino acids (Additional file 3: Figure S3), and a 2618 bp 3' UTR. According to the prediction of the ProtParam, the molecular mass of the goose AdipoR2 protein is $43.5533 \mathrm{kDa}$, and the theoretical isoelectric point is 5.6. The subcellular distribution of the AdipoR2 protein was predicted to be $65.2 \%$ in the plasma membrane, $26.1 \%$ in the endoplasmic reticulum, $4.3 \%$ in the Golgi, and $4.3 \%$ in the mitochondria. As expected, seven transmembrane domains were also found from the amino acid residues 145 to 167 , 180 to 202,217 to 239,246 to 265,275 to 297,310 to 332 , and 342 to 364 . Thirteen putative phosphorylation sites were identified in the AdipoR2 protein, which included 
ten serine residues (Ser12, Ser34, Ser38, Ser39, Ser52, Ser57, Ser58, Ser67, Ser215, and Ser220), one threonine residue (Thr374), and two tyrosine residues (Tyr119 and Tyr219). One putative N-glycosylation site located at amino acid positions 170 was identified. The secondary structure of the AdipoR2 protein was predicted to consist of $35.23 \% \alpha$-helix, $23.06 \%$ extended strand, $8.29 \% \beta$-turn, and $33.42 \%$ random coil.

\section{Sequence alignment and phylogenetic analysis}

The amino acid sequence similarities between Huoyan goose adiponectin, AdipoR1 and AdipoR2 and those of other representative species were investigated by multiple sequence alignment using the ClustalX2 program (See Additional file 4: Figure S4, Additional file 5: Figure S5 and Additional file 6: Figure S6). The overall percent identity among these sequences is shown in Table 2; the deduced amino acid sequence of Huoyan goose adiponectin shared $96 \%, 99 \%$, and $85 \%$ homology with duck (Anas platyrhynchos), chicken (Gallus gallus), and turkey (Meleagris gallopavo) adiponectin, respectively, and 70-73\% similarity with Sheep (Ovis aries), Dog (Canis lupus familiaris), Cat (Felis catus), human (Homo sapiens), turkey (Meleagris gallopavo), mouse (Mus musculus), pig (Sus scrofa). Unlike adiponectin, the deduced amino acid sequence of goose AdipoR1 and AdipoR2 are highly similar to that of mammalian AdipoR1 (91-99\%) and AdipoR2 (82-99\%).

A phylogenetic tree was constructed using the MEGA program based on the amino acid sequences of Huoyan goose adiponectin, AdipoR1 and AdipoR2 and the other species previously mentioned (See Additional file 7: Figure S7, Additional file 8: Figure S8 and Additional file 9: Figure S9). They were clustered into two subgroups consisting of avian species (including goose, duck, turkey, zebra finch, and chicken) in one group and

Table 2 Amino acid sequence identities of adiponectin, AdipoR1 and AdipoR2 between the Huoyan goose and other vertebrate species

\begin{tabular}{|c|c|c|c|}
\hline Gene & Matched species & GenBank accession no. & $\%$ Identity \\
\hline \multirow[t]{9}{*}{ adiponectin } & Duck (Anas platyrhynchos) & ADA68839.1 & 96 \\
\hline & Sheep (Ovis aries) & AHV91023.1 & 70 \\
\hline & Dog (Canis lupus familiaris) & BAD15362.1 & 71 \\
\hline & Cat (Felis catus) & BAF52934.1 & 73 \\
\hline & Chicken (Gallus gallus) & AAX40986.1 & 99 \\
\hline & Human (Homo sapiens) & NP_004788.1 & 73 \\
\hline & Turkey (Meleagris gallopavo) & XP_010714799.1 & 85 \\
\hline & Mouse (Mus musculus) & NP_033735.3 & 71 \\
\hline & Pig (Sus scrofa) & ABQ95350.1 & 71 \\
\hline \multirow[t]{9}{*}{ AdipoR1 } & Duck (Anas platyrhynchos) & $A B \mid 49513.2$ & 99 \\
\hline & Sheep (Ovis aries) & AHV91022.1 & 91 \\
\hline & Dog (Canis lupus familiaris) & XP_005622230.1 & 90 \\
\hline & Cat (Felis catus) & NP_001128153.1 & 90 \\
\hline & Chicken (Gallus gallus) & NP_001026198.1 & 97 \\
\hline & Human (Homo sapiens) & NP_001277558.1 & 91 \\
\hline & Zebra finch (Taeniopygia guttata) & XP_002198547.1 & 95 \\
\hline & Mouse (Mus musculus) & NP_082596.2 & 91 \\
\hline & Pig (Sus scrofa) & NP_001007194.1 & 91 \\
\hline \multirow[t]{9}{*}{ AdipoR2 } & Duck (Anas platyrhynchos) & ABC75392.1 & 99 \\
\hline & Bovine (Bos taurus) & NP_001035589.1 & 84 \\
\hline & Dog (Canis lupus familiaris) & XP_005637426 & 84 \\
\hline & Cat (Felis catus) & XP_011282054.1 & 83 \\
\hline & Chicken (Gallus gallus) & NP_001007855.1 & 97 \\
\hline & Human (Homo sapiens) & NP_078827.2 & 84 \\
\hline & Turkey (Meleagris gallopavo) & XP_003202489.1 & 97 \\
\hline & Mouse (Mus musculus) & NP_932102.2 & 84 \\
\hline & Pig (Sus scrofa) & NP_001007193.1 & 82 \\
\hline
\end{tabular}


mammalian species in the other. The phylogenetic tree indicated that the deduced goose adiponectin, and AdipoR1 and AdipoR2 proteins showed a closer genetic relationship to the avian species than to the mammal species.

\section{Expression profile of adiponectin, AdipoR1 and AdipoR2 mRNA in hypothalamus of Huoyan goose during different stages of the egg-laying cycle}

The mRNA levels of adiponectin, AdipoR1 and AdipoR2 in the hypothalamus of Huoyan geese during pre-laying, early-laying, peak-laying, and ceased periods were determined with qRT-PCR. As shown in Fig. 1, the expression of adiponectin mRNA increased from the pre-laying period to the peak-laying period, reached its peak during the peak-laying period, and then decreased and to the lowest level of expression during the ceased period. Conversely, the expression levels of AdipoR1 and AdipoR2 mRNA in the early-laying, peak-laying, and ceased periods were lower compared with the pre-laying period.

\section{Expression profile of adiponectin, AdipoR1 and AdipoR2 proteins in hypothalamus of Huoyan goose during different stages of the egg-laying cycle}

Western blotting was used to analyse the protein expression of adiponectin, AdipoR1 and AdipoR2 in the hypothalamus of Huoyan geese during different egg-laying stages. Similar to the qRT-PCR results, the expression level of the adiponectin protein was the highest during the peak-laying period from pre-laying period, and decreased in ceased period. The AdipoR1 and AdipoR2 protein concentrations were highest during pre-laying period (Fig. 2).

\section{Discussion}

Our study is the first to clone and characterize the cDNA and amino acid sequences of goose adiponectin and its receptors (AdipoR1 and AdipoR2) and compare the expression levels of these gene transcripts and proteins in the hypothalamus of Huoyan geese during different stages of the egg-laying cycle. We observed that adiponectin and its receptors were expressed in the hypothalamus tissues of Huoyan geese at both the mRNA and protein levels. Additionally the stage of the egg-laying cycle affected the abundance of mRNAs and proteins of both adiponectin and its receptors, which varied throughout the egg-laying cycle. We hypothesize these changes in expression might be related to the egglaying process in birds.

First, hormones and other physiological conditions including energy metabolism strictly control the eggproduction process in birds, from follicle maturation to ovulation. Moreover, it has long been recognized that female reproductive success depends largely on nutritional status and energy balance [24]. AMP-activated protein kinase (AMPK) is a master regulator of cellular and systematic energy homeostasis. Decreased AMPK activity in the hypothalamus leads to reduced food intake and increased energy expenditure [25]. Adiponectin was shown to stimulate fatty acid oxidation and enhance glucose uptake through the activation of AMP-activated protein kinase (AMPK) in the peripheral tissues, and activate AMPK via its receptor in the hypothalamus, stimulating food intake and decreasing energy expenditure [26, 27]. Recent studies suggest that increased expression of adiponectin depends on high nutritional status based on increased food intake [28]. Accordingly low circulating adiponectin levels were observed during the fasting period [29]. For avian species, adiponectin is likely to play a dominant role in carbohydrate and lipid metabolism because of the requirement to maintain a very high blood glucose concentration and lipid synthesis [30]. In our study, the varied expression level of adiponectin and its receptors throughout the egg-laying cycle may correlate with food availability and metabolic status during different egg-laying stages.

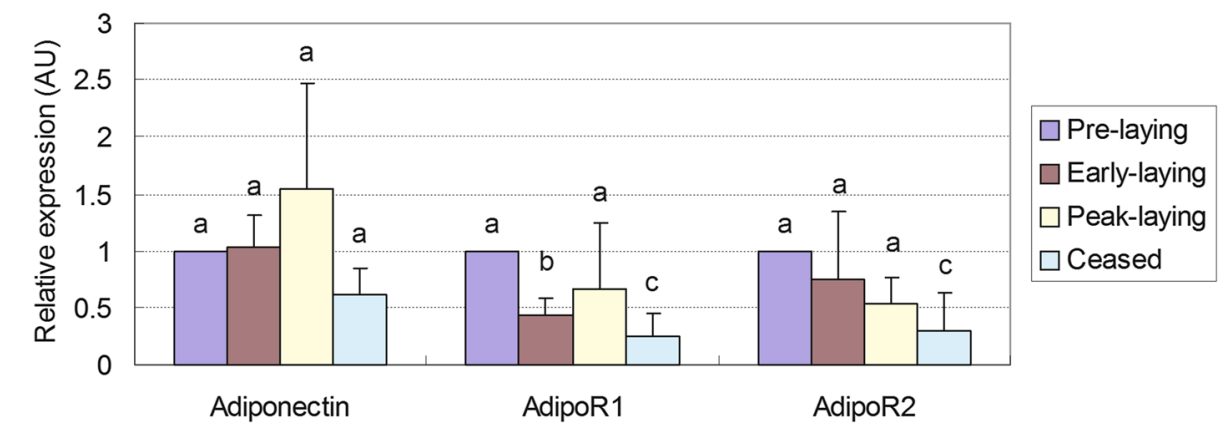

Fig. 1 Relative expression of adiponectin, AdipoR1 and AdipoR2 mRNA in the hypothalamus of Huoyan geese during different stages of the egglaying cycle. The expression levels of adiponectin, AdipoR1 and AdipoR2 were normalized to $18 \mathrm{~S}$ rRNA. The expression levels, calculated by the $2^{-\triangle \Delta C t}$ method, are presented as arbitrary units (AU). The presented values are the means \pm SEM. The data were analysed by ANOVA followed by Tamhane's T2 test post hoc test. Bars with different superscripts are significantly different $(P<0.05)$. 


\section{Pre-laying Early-laying Peak-laying Ceased

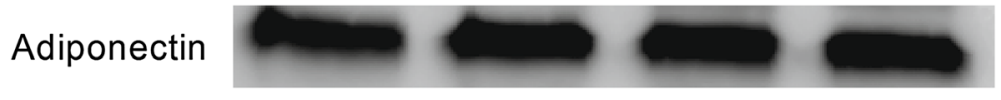

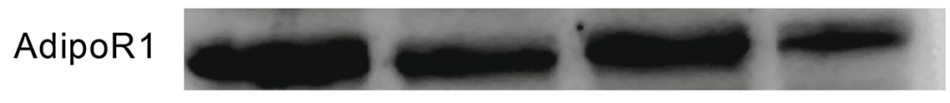
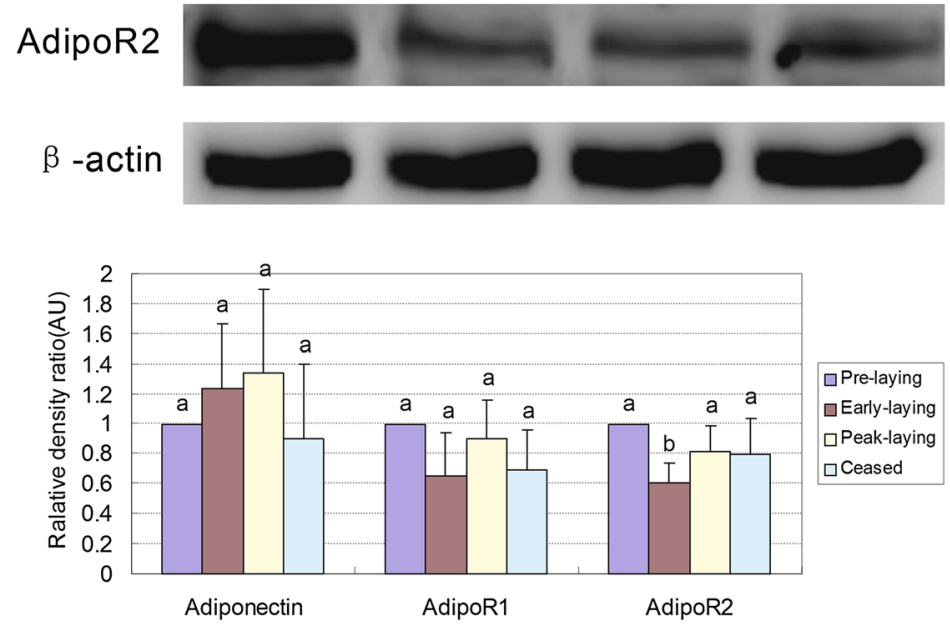

Fig. 2 Relative expressions of adiponectin, AdipoR1 and AdipoR2 protein in the hypothalamus of Huoyan geese during different stages of the egg-laying cycle. A comparison of adiponectin, AdipoR1 and AdipoR2 protein content were determined by western blotting analysis. Protein band density was analysed with GelQuant software. Beta-Actin was used as the internal control. Upper panels: representative immunoblots. Lower panels: densitometric analysis of adiponectin, AdipoR1 and AdipoR2 proteins relative to actin protein. Values are expressed as the mean \pm SEM of arbitrary optical density units. Data were analysed by ANOVA followed by Tamhane's T2 test post hoc test. Bars with different superscripts are significantly different $(P<0.05)$.

Second, laying performance is determined by the number of follicles destined for ovulation and the capacity of the oviduct to transform the ova into a hard-shelled egg. The development of follicles and ovulation are dependent on gonadotropins including luteinizing hormone (LH) and follicle-stimulating hormone (FSH) secreted by the pituitary, which in turn is regulated by the hypothalamic gonadotropin releasing hormones (GnRH) (Kuo et al., 2005). An increasing amount of evidence suggests that multiple hormones associated with reproductive processes could be responsible for the complex regulation of the adiponectin system. The presence of adiponectin and its receptors in the human, pig, rat, murine, and chicken hypothalamus and pituitary suggests that adiponectin may be a factor in modulating the secretory functions of the central branches of hormonal axes and, indirectly, also their peripheral branches, including the HPG axis [12-14, 18, 31, 32]. Adiponectin can exert autocrine/paracrine effects on GnRH synthesis and the pituitary secretory functions and may then indirectly affect gonadal functions. Adiponectin was found to regulate $\mathrm{GnRH}$ secretions by activating the AMPK pathway and inhibiting the ERK pathway in vivo [33] and was also observed to influence growth hormone and LH secretion in vitro by the isolated anterior pituitary cells of rats [13]. In addition, in isolated anterior (AP) and posterior (NP) pituitary cells of pigs during different stages of the estrous cycle, adiponectin affected GnRHand/or insulin-induced LH and FSH output in a manner dependent on the phase of the estrous cycle [34]. Furthermore, adiponectin also locally affects ovarian steroidogenesis and stimulated secretion of steroid hormones by regulating transport of total cholesterol, precursor for steroidogenesis in ovary. In adiponectin treated porcine granulosa cells from medium follicles of prepubertal gilts, higher levels of StAR and a lower expression of the P450 aromatase gene were observed [16]. Likewise, regulation of the expression of these steroidogenic genes (Cyp11a1, StAR, and Cyp19a1) by adiponectin also has been observed in the rat, bovine and human ovary $[17,35]$. These finding suggest that adiponectin might affect steroidogenesis in ovarian cells through the regulation of steroidogenic gene expressions as well. Notably, Anuradha et al. revealed that a low dose of adiponectin treatment caused a significant increase in circulating progesterone and oestradiol concentrations in C. sphinx, whereas a high dose of adiponectin caused a significant increase in oestradiol levels, but only a marginal increase in the progesterone level [36]. Edmond P. et al. demonstrated that adiponectin was able to augment the human Chorionic Gonadotrophin 
(hCG)-stimulated activity of the 3ßHSD enzyme and resulted in an acute increase in progesterone production, this suggested adiponectin may play a role in the rise in progesterone production approaching and following ovulation [5]. In chicken granulosa cells, adiponectin alone increased insulin-like growth factor 1 (IGF-1)-induced progesterone secretion, but in combination with $\mathrm{LH}$ or follicle-stimulating hormone (FSH), adiponectin reduced progesterone secretion in cultured granulosa cells [15]. We hypothesize that the egg-laying process of bird is regulated by a variety of reproductive hormones, and our findings suggest that the adiponectin system may affect egg-laying performance through changes in endocrine functions. Additionally the adiponectin system could also affect gonadal functions by controlling the secretory activity of the hypothalamus and pituitary. In our previous report, the mRNA expression of another gene, synaptotagmin-1 (syt1), was also found to change in a manner similar to adiponectin in the same animal experiment. As a major transducer of $\mathrm{Ca}^{2+}$ signalling for dense-core vesicle exocytosis in neuroendocrine cells, syt1 can regulate the secretion of neurotransmitters and hormones of the hypothalamus and pituitary, including those relevant to reproduction [37]. These consistent findings require further investigation to determine the mechanism of action.

Finally, the synthesis and expression of adiponectin and its receptors are probably hormonally controlled and correlated with the animals' hormonal milieu. Except for the expression inhibition of adiponectin mRNA by $\mathrm{GnRH}$ in immortalized LbT2 pituitary gonadotroph cells and primary pituitary cells of male rats, the progesterone level was found to be positively correlated with the adiponectin expression pattern [38]. Similar findings were reported by Caminos et al. who noted that progesterone had a stimulating effect on AdipoR2 gene expression [39]. Tan et al. observed that other gonadal steroids such as oestradiol and testosterone increase the mRNA and protein levels of the AdipoRs in cultured human adipocytes [40]. The reproductive cycle is controlled by FSH or LH, in bovine theca interna cells, and LH increased the concentrations of AdipoR2 mRNAs, and may be involved in the regulation of adiponectin receptor expression[17]. In addition, adiponectin may conduct its action through up- and/or downregulation of its own receptors, and could be an important factor in modulating its own receptor levels. Rodriguez-Pacheco et al. observed a significant decrease in AdipoR1 mRNA levels and an increase in AdipoR2 mRNA concentrations was noted in cultures of rat pituitary cells exposed to $10^{-8} \mathrm{M}$ and $10^{-7} \mathrm{M}$ of adipokine for $24 \mathrm{~h}$, respectively [13]. Contrary results were presented by Caminos et al. who observed a suppressive effect of adiponectin on AdipoR2 mRNA expression in cultured human placenta explants
[39]. Similar to the aforementioned findings, our study revealed the differential expression of adiponectin and its receptors from the pre-laying period to the ceased period. One possible explanation for this difference in accordance with egg-laying performance may be due to changes in sex hormone levels throughout the egg-laying cycle.

Collectively, the varied expression profiles of adiponectin, AdipoR1 and AdipoR2 suggests that adiponectin improves glucose utilization and regulates energy balance in response to nutritional states of the whole-body during different stages of the egg-laying cycle and subsequently determines the release of GnRH or gonadotropin to influence reproduction. The idea that the adiponectin system may function in the ovary during steroidogenesis and maturation of the oocyte is also supported.

\section{Conclusions}

Our study was the first experiment to demonstrate the presence of both the mRNA and protein of adiponectin and its receptors in the Huoyan goose hypothalamus, and the effect of different stages of the egg-laying cycle on their expression. The variations in the expression levels of adiponectin and its receptor in the hypothalamus could be attributed to the influence of multiple hormones controlling reproductive processes. The results of this study suggested a role for adiponectin as a key neuromodulator of reproductive functions, which might operate as an endocrine integrator linking metabolism and gonadal function.

\section{Additional files}

\begin{abstract}
Additional file 1: Figure S1. Nucleotide and deduced amino acid sequences of adiponectin. The nucleotide (black) and deduced amino acid (blue) sequences are shown and numbered on the left. The nucleotide sequence is numbered from the $5^{\prime}$ end. The first methionine (M) is the first deduced amino acid. The C1Q domain (amino acids 105-241) is shaded. The start codons (ATG) and the stop codons (TAA) are marked in bold red. (TIFF 315 kb)
\end{abstract}

Additional file 2: Figure S2. Nucleotide and deduced amino acid sequences of AdipoR1. The nucleotide (black) and deduced amino acid (blue) sequences are shown and numbered on the left. The nucleotide sequence is numbered from the $5^{\prime}$ end. The first methionine $(M)$ is the first deduced amino acid. The start codons (ATG) and the stop codons (TAA) are marked in bold red. (TIFF $727 \mathrm{~kb}$ )

Additional file 3: Figure S3. Nucleotide and deduced amino acid sequences of AdipoR2. The nucleotide (black) and deduced amino acid (blue) sequences are shown and numbered on the left. The nucleotide sequence is numbered from the $5^{\prime}$ end. The first methionine $(M)$ is the first deduced amino acid. The start codons (ATG) and the stop codons (TAA) are marked in bold red. (TIFF $741 \mathrm{~kb}$ )

Additional file 4: Figure S4. Multiple amino acid sequence alignment of the Huoyan goose adiponectin protein with other vertebrate species. The colour black denotes $100 \%$ conserved sequences, and the colour grey indicates non-conservative sequences. Gaps (-) were introduced to maximize the alignment. Sequences for the alignment were obtained from GenBank (accession numbers are in brackets): Anas platyrhynchos (ADA68839.1); Ovis aries (AHV91023.1); Canis lupus familiaris (BAD15362.1); Felis catus (BAF52934.1); Gallus (AAX40986.1); Homo sapiens 
(NP_004788.1); Meleagris gallopavo (XP_010714799.1); Mus musculus (NP_033735.3); and Sus scrofa (ABQ95350.1). (TIFF $3483 \mathrm{~kb}$ )

Additional file 5: Figure S5. Multiple amino acid sequence alignment of the Huoyan goose AdipoR1 protein with other vertebrate species. The colour black denotes $100 \%$ conserved sequences, and the colour grey indicates non-conservative sequences. Gaps (-) were introduced to maximize the alignment. Sequences for the alignment were obtained from GenBank (accession numbers are in brackets): Anas platyrhynchos (ABI49513.2); Ovis aries (AHV91022.1); Canis lupus familiaris (XP_005622230.1); Felis catus (NP_001128153.1); Gallus (NP_001026198.1); Homo sapiens (NP_001277558.1); Taeniopygia guttata (XP_002198547.1); Mus musculus (NP_082596.2); and Sus scrofa (NP_001007194.1). (TIFF $4230 \mathrm{~kb})$

Additional file 6: Figure S6. Multiple amino acid sequence alignment of the Huoyan goose AdipoR2 protein with other vertebrate species. The colour black denotes $100 \%$ conserved sequences, and the colour grey indicates non-conservative sequences. Gaps (-) were introduced to maximize the alignment. Sequences for the alignment were obtained from GenBank (accession numbers are in brackets): Anas platyrhynchos (ABC75392.1); Bos taurus (NP_001035589.1); Canis lupus familiaris (XP_005637426); Felis catus (XP_011282054.1); Gallus (NP_001007855.1); Homo sapiens (NP_078827.2); Meleagris gallopavo (XP_003202489.1); Mus musculus (NP_932102.2); and Sus scrofa (NP_001007193.1). (TIFF $5171 \mathrm{~kb}$ )

Additional file 7: Figure S7. The phylogenetic tree of adiponectin protein was constructed using the neighbour-joining method with MEGA4. Amino acid sequences of adiponectin for these species were downloaded from the protein database of the NCBI. Their corresponding accession numbers are the same as those given in Table 2 . The number at the branches denotes the bootstrap majority consensus values on 1000 replicates; the branch lengths represent the relative genetic distances among these species. (TIFF $7463 \mathrm{~kb}$ )

Additional file 8: Figure S8. The phylogenetic tree of AdipoR1 protein was constructed using the neighbour-joining method with MEGA4. The amino acid sequences of adiponectin for these species were downloaded from the NCBI protein database. Their corresponding accession numbers are shown in Table 2. The number at the branches denotes the bootstrap majority consensus values on 1000 replicates; the branch lengths represent the relative genetic distances among these species. (TIFF $7504 \mathrm{~kb}$ )

Additional file 9: Figure S9. The phylogenetic tree of the AdipoR2 protein was constructed using the neighbour-joining method with MEGA4. The amino acid sequences of adiponectin for these species were downloaded from the NCBI protein database. Their corresponding accession numbers are the same as those given in Table 2 . The number at the branches denotes the bootstrap majority consensus values on 1000 replicates; the branch lengths represent the relative genetic distances among these species. (TIFF $7477 \mathrm{~kb}$ )

\section{Abbreviations}

aa: Amino acid(s); bp: Base pair(s); cDNA: DNA complementary to RNA; kDa: Kilodalton; mRNA: Messenger RNA; ORF: Open reading frame; PCR: Polymerase chain reaction; RACE: Rapid-amplification of CDNA ends; rRNA: Ribosomal RNA; UTR: Untranslated Regions.

\section{Competing interests}

The authors declare that they have no competing interests.

\section{Author contributions}

$X L$ designed the study. $Z C, J L, L L$ and $S L$ performed the experiments and data analysis. ML and MG contributed reagents, materials, and analysis tools. ZC and XL wrote the manuscript. HY revised the manuscript. All authors read and approved the final manuscript.

\section{Acknowledgements}

This study was supported by the National Natural Science Foundation of China (Grant No. 31172286). We would like to thank the staff of Liaoning Huoyan Goose Stock Breeding Farm, who assisted in the collection of the goose hypothalamus samples. We also acknowledge Dr. Sarah S. at the American Journal Experts (AJE), for her editing and polish to improve the manuscript.

\section{Author details}

${ }^{1}$ College of Animal Science and Veterinary Medicine, Shenyang Agricultural University, Shenyang 110866, China. ${ }^{2}$ Guizhou Minzu University, Guiyang 550025, China.

Received: 20 May 2015 Accepted: 29 July 2015

Published online: 07 August 2015

\section{References}

1. Scherer PE, Williams S, Fogliano M, Baldini G, Lodish HF. A novel serum protein similar to $\mathrm{Clq}$, produced exclusively in adipocytes. J Biol Chem. 1995;270:26746-9.

2. Pajvani UB, Du X, Combs TP, Berg AH, Rajala MW, Schulthess T, et al. Structure-function studies of the adipocyte-secreted hormone Acrp30/ adiponectin. Implications fpr metabolic regulation and bioactivity. J Biol Chem. 2003;278:9073-85.

3. Chabrolle C, Tosca L, Crochet S, Tesseraud S, Dupont J. Expression of adiponectin and its receptors (AdipoR1 and AdipoR2) in chicken ovary: potential role in ovarian steroidogenesis. Domest Anim Endocrinol. 2007;33:480-7.

4. Yamauchi T, Kamon J, Ito Y, Tsuchida A, Yokomizo T, Kita S, et al. Cloning of adiponectin receptors that mediate antidiabetic metabolic effects. Nature. 2003;423:762-9.

5. Wickham 3rd EP, Tao T, Nestler JE, McGee EA. Activation of the LH receptor up regulates the type 2 adiponectin receptor in human granulosa cells. J Assist Reprod Genet. 2013;30:963-8.

6. Ramachandran R, Maddineni S, Ocon-Grove O, Hendricks 3rd G, VasilatosYounken R, Hadley JA. Expression of adiponectin and its receptors in avian species. Gen Comp Endocrinol. 2013;190:88-95.

7. Kaminski T, Smolinska N, Maleszka A, Kiezun M, Dobrzyn K, Czerwinska J, et al. Expression of adiponectin and its receptors in the porcine hypothalamus during the oestrous cycle. Reprod Domest Anim. 2014:49:378-86.

8. Smolinska N, Dobrzyn K, Maleszka A, Kiezun M, Szeszko K, Kaminski T. Expression of adiponectin and adiponectin receptors 1 (AdipoR1) and 2 (AdipoR2) in the porcine uterus during the oestrous cycle. Anim Reprod Sci. 2014;146:42-54.

9. Wen JP, Lv WS, Yang J, Nie AF, Cheng XB, Yang Y, et al. Globular adiponectin inhibits $\mathrm{GnRH}$ secretion from GT1-7 hypothalamic GnRH neurons by induction of hyperpolarization of membrane potential. Biochem Biophys Res Commun. 2008;371:756-61.

10. Kiezun M, Maleszka A, Smolinska N, Nitkiewicz A, Kaminski T. Expression of adiponectin receptors 1 (AdipoR1) and 2 (AdipoR2) in the porcine pituitary during the oestrous cycle. Reprod Biol Endocrinol. 2013;11:18.

11. Hoyda TD, Fry M, Ahima RS, Ferguson AV. Adiponectin selectively inhibits oxytocin neurons of the paraventricular nucleus of the hypothalamus. J Physiol. 2007:585:805-16.

12. Psilopanagioti A, Papadaki H, Kranioti EF, Alexandrides TK, Varakis JN Expression of adiponectin and adiponectin receptors in human pituitary gland and brain. Neuroendocrinology. 2009;89:38-47.

13. Rodriguez-Pacheco F, Martinez-Fuentes AJ, Tovar S, Pinilla L, Tena-Sempere M, Dieguez C, et al. Regulation of pituitary cell function by adiponectin. Endocrinology. 2007;148:401-10.

14. Steyn FJ, Boehme F, Vargas E, Wang K, Parkington HC, Rao JR, et al. Adiponectin regulate growth hormone secretion via adiponectin receptor mediated $\mathrm{Ca}^{2+}$ signalling in rat somatotrophs in vitro. J Neuroendocrinol. 2009:21:698-704

15. Chabrolle C, Tosca L, Crochet S, Tesseraud S, Dupont J. Expression of adiponectin and its receptors (AdipoR1 and AdipoR2) in chicken ovary: Potential role in ovarian steroidogenesis. Domest Anim Endocrinol. 2007:33:480-7

16. Ledoux S, Campos DB, Lopes FL, Dobias-Goff M, Palin MF, Murphy BD. Adiponectin induces periovulatory changes in ovarian follicular cells. Endocrinology. 2006;147:5178-86.

17. Lagaly DV, Aad PY, Grado-Ahuir JA, Hulsey LB, Spicer LJ. Role of adiponectin in regulating ovarian theca and granulosa cell function. Mol Cell Endocrinol. 2008:284:38-45.

18. Kiezun M, Maleszka A, Smolinska N, Nitkiewicz A, Kaminski T. Expression of adiponectin receptors 1 (AdipoR1) and 2 (AdipoR2) in the porcine pituitary during the oestrous cycle. Reprod Biol Endocrinol. 2013;11:18. 
19. Tabandeh MR, Hosseini A, Saeb M, Kafi M, Saeb S. Changes in the gene expression of adiponectin and adiponectin receptors (AdipoR1 and AdipoR2) in ovarian follicular cells of dairy cow at different stages of development. Theriogenology. 2010;73:659-69.

20. Padmanabhan V, Karsch FJ, Lee JS. Hypothalamic, pituitary and gonadal regulation of FSH. Reprod Suppl. 2002;59:67-82.

21. Larkin MA, Blackshields G, Brown NP, Chenna R, McGettigan PA, McWilliam $H$, et al. Clustal W and Clustal X version 2.0. Bioinformatics. 2007;23:2947-8.

22. Tamura K, Dudley J, Nei M, Kumar S. MEGA4: Molecular Evolutionary Genetics Analysis (MEGA) software version 4.0. Mol Biol Evol. 2007;24:1596-9.

23. Livak KJ, Schmittgen TD. Analysis of relative gene expression data using real-time quantitative PCR and the 2(-Delta Delta C(T)) Method. Methods. 2001:25:402-8.

24. Garcia-Garcia RM. Integrative control of energy balance and reproduction in females. ISRN Vet Sci. 2012;2012:121389.

25. Kim MS, Lee KU. Role of hypothalamic 5'-AMP-activated protein kinase in the regulation of food intake and energy homeostasis. J Mol Med (Berl). 2005;83:514-20.

26. Kubota N, Yano W, Kubota T, Yamauchi T, Itoh S, Kumagai H, et al. Adiponectin stimulates AMP-activated protein kinase in the hypothalamus and increases food intake. Cell Metab. 2007;6:55-68.

27. Guillod-Maximin E, Roy AF, Vacher CM, Aubourg A, Bailleux V, Lorsignol A, et al. Adiponectin receptors are expressed in hypothalamus and colocalized with proopiomelanocortin and neuropeptide $Y$ in rodent arcuate neurons. J Endocrinol. 2009;200:93-105.

28. Cahill F, Amini P, Wadden D, Khalili S, Randell E, Vasdev S, et al. Short-term overfeeding increases circulating adiponectin independent of obesity status. PLoS One. 2013;8, e74215.

29. Kubota N, Terauchi Y, Yamauchi T, Kubota T, Moroi M, Matsui J, et al. Disruption of adiponectin causes insulin resistance and neointimal formation. J Biol Chem. 2002;277:25863-6.

30. Brady LJ, Romsos DR, Brady PS, Bergen WG, Leveille GA. The effects of fasting on body composition, glucose turnover, enzymes and metabolites in the chicken. J Nutr. 1978;108:648-57.

31. Lord E, Ledoux S, Murphy BD, Beaudry D, Palin MF. Expression of adiponectin and its receptors in swine. J Anim Sci. 2005;83:565-78.

32. Maddineni S. Adiponectin Gene Is Expressed in Multiple Tissues in the Chicken: Food Deprivation Influences Adiponectin Messenger Ribonucleic Acid Expression. Endocrinology. 2005;146:4250-6.

33. Cheng XB, Wen JP, Yang J, Yang Y, Ning G, Li XY. GnRH secretion is inhibited by adiponectin through activation of AMP-activated protein kinase and extracellular signal-regulated kinase. Endocrine. 2011;39:6-12.

34. Kiezun M, Smolinska N, Maleszka A, Dobrzyn K, Szeszko K, Kaminski T. Adiponectin expression in the porcine pituitary during the estrous cycle and its effect on LH and FSH secretion. Am J Physiol Endocrinol Metab. 2014;307:E1038-1046.

35. Chabrolle C, Tosca L, Dupont J. Regulation of adiponectin and its receptors in rat ovary by human chorionic gonadotrophin treatment and potential involvement of adiponectin in granulosa cell steroidogenesis. Reproduction. 2007;133:719-31.

36. Anuradha, Krishna A. Modulation of ovarian steroidogenesis by adiponectin during delayed embryonic development of Cynopterus sphinx. J Steroid Biochem Mol Biol. 2014;143:291-305.

37. Luan X, Luo L, Cao Z, Li R, Liu D, Gao M, et al. Molecular cloning and expression analysis of the Synaptotagmin-1 gene in the hypothalamus and pituitary of Huoyan goose during different stages of the egg-laying cycle. Reprod Biol Endocrinol. 2014;12:83.

38. Kim J, Zheng W, Grafer C, Mann ML, Halvorson LM. GnRH decreases adiponectin expression in pituitary gonadotropes via the calcium and PKA pathways. Reprod Sci. 2013;20:937-45.

39. Caminos JE, Nogueiras R, Gallego R, Bravo S, Tovar S, Garcia-Caballero T, et al. Expression and regulation of adiponectin and receptor in human and rat placenta. J Clin Endocrinol Metab. 2005;90:4276-86.

40. Tan BK, Chen J, Digby JE, Keay SD, Kennedy CR, Randeva HS. Upregulation of adiponectin receptor 1 and 2 mRNA and protein in adipose tissue and adipocytes in insulin-resistant women with polycystic ovary syndrome. Diabetologia. 2006;49:2723-8

\section{Submit your next manuscript to BioMed Central and take full advantage of:}

- Convenient online submission

- Thorough peer review

- No space constraints or color figure charges

- Immediate publication on acceptance

- Inclusion in PubMed, CAS, Scopus and Google Scholar

- Research which is freely available for redistribution

Submit your manuscript at www.biomedcentral.com/submit 